\title{
Enhancement Of Spatial Structure Of An Image By Using Texture Feature Extraction
}

\author{
Turkan Ahmed Khaleel \\ Department of Computer Engineering \\ College of Engineering - Mosul University
}

\begin{abstract}
In this paper a new algorithm has been developed to enhance spatial structure by using texture feature extraction. Then, this algorithm has been programmed and an integrated program has been prepared by using Microsoft Visual $\mathrm{C}++6.0$. In order to ascertain the usefulness of this algorithm, it has been applied in two groups of images, the first group consisted of three images of different feature. The results have been evident enhancement in spatial; structure. The second group of images consisted of multi-spectral images taken by Thematic Mapper (TM) in the city of Mosul. The results of this algorithm have been used in the classification.

The measurement of classification accuracy of the results of this algorithm compared with the original images which have not undergone any spatial structure enhancement revealed that this algorithm executed the process of texture feature extraction excellently. Further, the results were clear in the barren areas, Agricultural fields and the other areas as well.
\end{abstract}

Keywords: texture feature extraction, Spatial filter, Classification

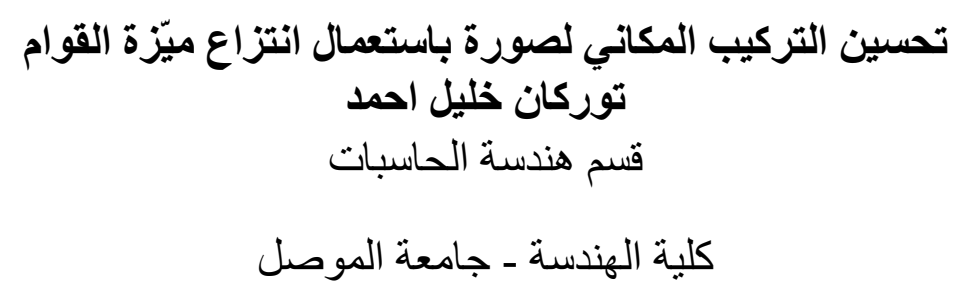




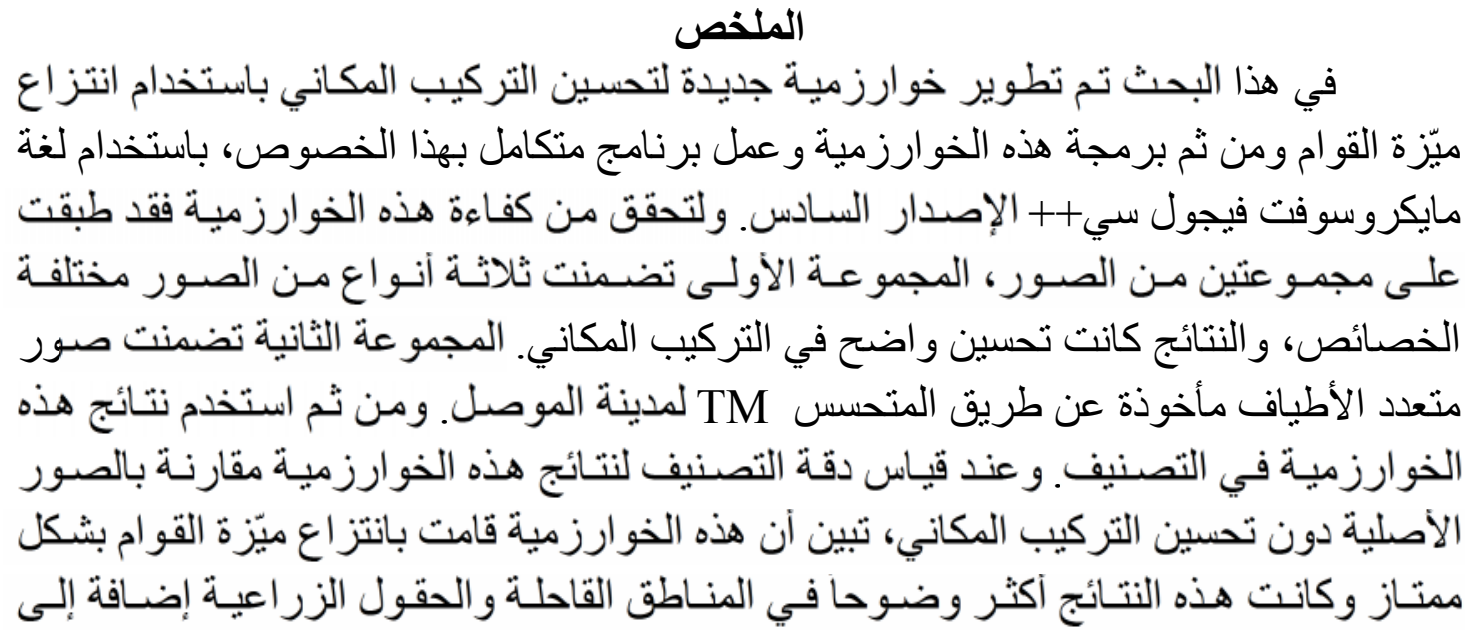

\section{1-Reteaductionov. 2005}

Accepted 30 April

- - The main objective of enhancement techniques is to work out a certain image on the condition that the result would be more suitable than the original image. A number of techniques used to improve digital image are available. These are known as image enhancement procedures [1].

The techniques of image enhancement can be classified into three broad categories, namely: contrast manipulation, multi-image manipulation and spatial feature manipulation [2]. The present study is concerned with the last category; sometimes known as spatial enhancement.

Enhancement of spatial structure tries to improve the image by treating image's spatial frequency which implies a number of changes, brightness values per unit distance for any particular part of an image [3]. Through this type, it is possible to improve brightness value of a particular point depending on the brightness value of the neighboring point. Accordingly, this type of enhancement is also called area operation [4].

Spatial filtering is one way in which an image's spatial frequency can be enhanced. Convolution filtering is a type of spatial filtering in 
which a matrix of varying sizes is passed over an image and used to compute a central pixel value based on the neighboring pixel value [5]. For most filter types, the filter window is square with odd number of lines and columns, ranging from 3 by 3 to 11 by 11 .

A great number of filtering leads to texture feature extraction. Bovik et al., [6] presented texture as irradiance patterns that are distinguished on the basis of high concentration of localized spatial frequencies. Gong et al., [7] found that first order statistics like standard deviation is highly correlated to variance, it requires less computation time and can be successfully used as texture measure in classification. Other researchers depended on grey level co-occurrence matrix (GLCM). Baraldi and Parmiggiani [8] investigated the statistical meaning of six GLCM: energy, contrast, variance, correlation, entropy, and inverse difference. They concluded that the energy and contrast are considered to be the most efficient for discriminating different texture patterns, whereas Cruz and Dorronsor, [9] presented algorithm for a nonlinear supervised feature extraction containing Fisher's criterion with preliminary perceptron-like nonlinear projection of vectors in pattern space.

Randen and Husoy [10] presented an extensive study of different filtering theories which leads to texture extraction. The study included a comparison of different methods: Law's mask, ring/wedge filters, dyadic Gabor filter banks, wavelet transforms, wavelet packets and frames, Quadrature Mirro filters (QMF), Discrete consin Transform (DCT), eigen filters, optimized Gabor filters, linear predictors, and optimized finite impulse response filters. Researchers think that the simple hypothesis of most filtering theories is based on energy distribution in frequency domain and therefore, the spectral energy signatures for these are very different for different textures.

Studies have continued and greatly developed in this field. Battacharya et al., [11] developed the cross-diagonal texture filtering technique using two different approaches, namely, mean and median, and 
studied its suitability in eliminating noise in satellite remote sensing data. Grigoress et al., [12] presented a comparison between texture extractions based on Gabor filters. Rivero and Bres [13] studied texture discrimination depending on two families of filters, Gabor and Hermite. The study basically aims at developing a new algorithm enhancing spatial structure of an image by using a new technique for texture feature extraction. Puig and Garcia [14] added a new technique for selecting an alternative group for texture methods by being integrated in a more suitable way, especially if they belong to the same family. They also revealed that the experimental results in their true complicated image showed that the open selection scheme was more useful than the algorithm of selecting texture extraction.

For this purpose, an integrated program has been designed by using Microsoft Visual C++ 6.0. The program has been applied on different types of images, and the results were satisfying. The same program has been used to enhance the spatial structure of the image's remote sensing. The results then have been enhanced and classified. Maximum Likelihood, for example, is one of the well-known conventional methods for classifying images [15]. Using this type of classification with algorithm has been more accurate than using the original images in the classification without any enhancement.

The following is an explanation of the rules and algorithms used in the present study.

\section{2-Method and Algorithm}

Many spatial filters are implemented with convolution mask, because a convolution mask operation provides a result that is a weight sum of values of a pixel and its neighbors. One interesting aspect of convolution mask is that the overall effect can be predicated based on their general pattern [4]. 
If we call the convolution mask, $\operatorname{MASK}(\mathrm{x}, \mathrm{y})$ and the input image, $\operatorname{IMAGE}(\mathrm{r}, \mathrm{c})$, the convolution equation is given by:

$$
\operatorname{OUT}(r, c)=\sum_{x=0}^{N-1} \sum_{y=0}^{N-1} \operatorname{IMAGE}(r-x, c-y) \operatorname{MASK}(x, y)
$$

where:

OUT $(r, c)$ : is the output image.

$\operatorname{IMAGE}(r, c)$ : is the input image.

$\operatorname{MASK}(\mathrm{x}, \mathrm{y})$ : is the mask window.

$\mathrm{N}$ : is the mask window size (odd number).

$\mathrm{r}$ : is the row index.

c: is the column index.

In order to reduce calculating processes and execution time, the mask used in this study is an implied one; it can be used but it does not have a place as a variable in the computer memory. This process helps in reducing the execution cost. As for the mask coefficients, as shown in figure (1), they are simply pixel values(GL) taken from the original image. 


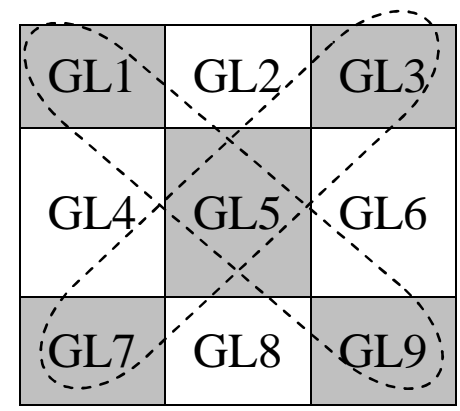

Figure(1): Mask coefficients and their calculation method

The figure explains mask coefficients calculation in this type of filter. The new pixel value which can be replaced in the resulting image, can be calculated according to the following equation:

Let $\mathrm{X}=\mathrm{GL} 1+\mathrm{GL} 5+\mathrm{GL} 9$

$$
\therefore \quad X=\sum_{i=0}^{N-1} f(i, i)
$$

Let $\mathrm{Y}=\mathrm{GL} 7+\mathrm{GL} 5+\mathrm{GL} 3$

$$
\therefore \quad Y=\sum_{i=0}^{N-1} f(N-1+i, i)
$$

$$
O U T(r, c)=\sqrt{X^{2}+Y^{2}}
$$


For further explanation of the idea of filter, the following is survey of the algorithm of this type of filter:

\begin{tabular}{|} 
Begin Algorithm \\
For $r=0$ To Wigth- 1 \\
For $c=0$ To High- 1 \\
Begin \\
$X=0$ \\
$Y=0$ \\
For $m=0$ To $N-1$
\end{tabular}

\section{3-Results and Discussion}

In this study, the image's spatial structure has been enhanced by developing a new algorithm for texture feature extraction after putting forward the algorithm of this filter. Further, an integrated program has been designed by using Microsoft Visual $\mathrm{C}++6.0$ which works under the system of windows as shown in Plate(1).

In this study, two groups of images have been chosen to insure the ability of this algorithm. The first group consisted of three images of different sizes and features. These are: the image of Water-Ice image $(200 \times 200)$ pixel, Fancy image $(99 \times 99)$ pixel and Wave image 
$(99 \times 99)$ pixel, as shown in Plate(2). The images were set in the program and after applying the suggested filter on each image, a fair amount of results have been arrived at as explained in Plate(3) where it is possible to notice an evident enhancement in spatial structures.

The second group of images consisted of multi-spectral images used in remote sensing and representing Mosul region. This group has been chosen to attach importance on the process of texture in analyzing and classifying the images. Plate(4) explains the three bands related to the sensor TM of the satellite Lansat5 with resolution $30 \mathrm{~m}$. Three bands were used: TM3 (0.63-0.69) $\mu \mathrm{m}$, TM5 (1.55-1.75) $\mu \mathrm{m}$, TM7(2.08$2.35) \mu \mathrm{m}$. The other bands have not been entered as they were not available at the time of preparing this study. Figure (2) shows the sequence of the processes conducted on the multi-spectral images.

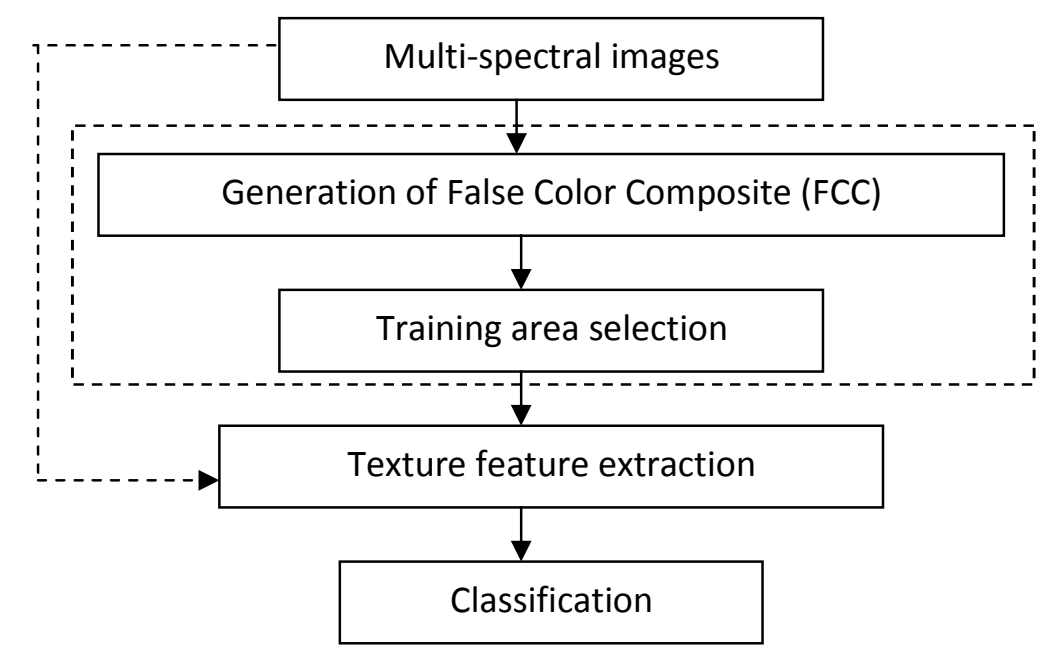

Figure 2: The steps of combination texture with spectral information in the classification of multi-spectral images. 
The first step includes reading multi-spectral images and setting them in the program. The second step is a matter of composing and displaying false color composite to choose training area and then to store them in a file to be used in classification then after.

According to the nature of the area and visual interpretation, six different classes have been chosen including: Rive, Lake, Canal; Urban Area; Grasses; Agricultural fields; Barren Area and Hill Area.

In the stage of texture feature extraction, the filter suggested in this study has been applied on multi-spectral images. Plate(5) shows the results of spatial structure enhancement of three bands, it can be noticed how urban areas were covered by the structures. These structures could possibly include cities, town, villages, roads and others. In order to emphasize this second part of the study which shows the high value of texture especially when combined with spectral information, the results of this stage have been entered into a classifier.

In the stage of classification, there exist several methods of classification [16-18]. However, the present study is not concerned with choosing a specifies classification method, it rather puts emphasis on using the texture in the classification. For this reason, it can be chosen the most conventional method, maximum likelihood [15].

Plate(6) shows a clear comparison of the results of the classification of multi-spectral images whose spatial structure has been enhanced by the new method of extracting texture feature together with the results of classifying original multi-spectral images which have not undergone any enhancement. Through the comparison, it can be noticed that urban areas considered to be among important spatial structures, have been classified in a good way. The classification also included other structures such as water area and Barren area.

The above explanation is relates to optic explanation. As far as the measurement of classification accuracy is considered, Table1, reveals 
classification accuracy of the six selected classes as well as overall accuracy. It is noticed that the classification overall accuracy of the original image $82.20 \%$ rose into $98.71 \%$ when we carried out the process of enhancement spatial structure of multi-spectral images by using the new filter before entering them in the classifier. The accuracy of each class especially the fifth class which represents Barren area 13.27 which cannot be separated from other classes such as the Urban area, Grasses and Agricultural fields was very low and rose into 98.37 when we used the suggested method.

Finally, the development of method and the algorithm of the filters of image's spatial structure enhancement and the program design are considered a positive process whose importance suits with information values which can be extracted from the image.

Table 1: The accuracy of individual classes, overall accuracy using maximum likelihood (ML) classification of original bands and classification bands after enhancement of spatial structure

\begin{tabular}{|l|c|c|}
\hline \multicolumn{1}{|c|}{ Individual } & $\begin{array}{l}\text { ML } \\
\text { classification } \\
\text { Accuracy } \\
\text { of original } \\
\text { bands(\%) }\end{array}$ & $\begin{array}{l}\text { ML } \\
\text { classification } \\
\text { of bands after } \\
\text { texture feature } \\
\text { extraction(\%) }\end{array}$ \\
\hline River ,Lake, Canal & 99.69 & 100.00 \\
\hline Urban Area & 99.73 & 99.94 \\
\hline Grasses & 96.42 & 98.48 \\
\hline Agricultural Fields & 85.34 & 95.51 \\
\hline Barren Area & 13.27 & 98.37 \\
\hline
\end{tabular}




\begin{tabular}{|l|c|c|}
\hline Hill Area & 98.77 & 100.00 \\
\hline Overall accuracy(\%) & $82.20 \%$ & $98.71 \%$ \\
\hline
\end{tabular}

\section{4-Conclusions}

The concern about the development of methods and algorithms of spatial structure enhancement by texture feature extraction is an important issue that suits with the importance of information hold by the texture. The texture is an innate quality in the bodies. It contains highly important information about the surfaces' skeleton structure. For this reason, a new algorithm enhancing the spatial structure by using a new type of filters of texture feature extraction, has been developed. The results have been satisfying. The combination of texture with spectral information after enhancing the spatial structure extraction from multispectral images and using them in analyzing and classifying the images regardless of the quality of the classifier used, leads to raise the performance to level of enhancement and classification accuracy in accordance with the results arrived at in this study.

\section{References}

1- Umbaugh S. E., Computer Vision and Image Processing: A practical Approach Using CVIP tools, Prentice-Hall, Inc., Asimon \& Schuster Company, 1998.

2- Lillesand T. M. and Kiefer R. W., Remote Sensing and Image Interpretation, $3^{\text {rd }}$ edition. New York, John Wiley and Sons, Inc, 1994.

3- Jensen, J. R., Introductory Digital Image Processing: A Remote Sensing Perspective, $2^{\text {nd }}$ edition, Englewood Cliffs, New Jersey, prentice-Hall, 1996, pp.153-172. 
4- Mather P. M., Computer Processing of Remote Sensing Images; An Introduction, John Wiley \& Sons, 1987.

5- Gonzalez, R. C. and Wintz, P., Digital Image Processing, $2^{\text {nd }}$ edition, Reading Messachuetts, Addison-Wesley publishing company, 1987, pp.140-203.

6- Bovik A. C., Clark M. and Geisler W. S., Multi channel Texture Analysis using Localized Spatial Filter, IEEE Transactions Pattern Analysis and Machine Intelligence, Vol. 12, No. 1, 1990, pp.55-73.

7- Gong P., Marceau J. D. and Howarth P. J., A Comparison of Spatial Feature Extraction Algorithms for Land-use Classification with SPOT HRV Data, Remote Sensing Environ., Vol. 40, 1992, pp.137151.

8- Baraldi A. and Parmiggiani F., An Investigation of the Textural Characteristics Associated with Gray Level Co occurrence Matrix Statistical parameters, IEEE Transaction on Geoscience and Remote Sensing, Vol. 33, No. 2, 1995, pp.293-304.

9- Cruze C. S. and Dorronsor J. R., A Nonlinear Discriminate Algorithm for Feature Extraction and Data Classification, IEEE Transactions on Neural Networks, Vol. 9, No. 6, 1998, pp.1370-1375.

URL: http://www.crisp.nus.edu.sg/ acrs2001/pdf/132bhatt.pdf

10- Randen T. Husoy J. H., Filtering for Texture Classification: A Comparative Study, IEEE Transaction on Pattern Analysis and Machine Intelligence, Vol. 21, No. 4, 1999, pp.291-310.

11- Battacharya A. K., Srivastava P. K. and Bhagat A., A Modified Texture Filtering Technique for Satellite Images, Center for Remote Imaging, Sensing and Processing(CRISP), 2001.

12- Grigoressu, S.E., Petkov, N. and Kruizinga P., Comparison of Texture Features Based on Gabor Filters, IEEE Transaction on Image Processing, Vol. 11, No. 10, 2002, pp.1160-1167.

13- Rivero-Moreno, C. J. and Bres S., Texture Analysis and Indexing using Gabor-Like Hermite Filters, submitted to Image and Vision Computing, 2004, URL: http://www.liris.cnrs.fr/docs/RP-2004028.dbf

14- Puig D. and Garcia M. A., Automatic Selection of Multiple Texture Feature Extraction Methods for Texture Pattern Classification, Springer-Verlag Berlin Heidelberg, 2005, pp.215-222.

15- Abkar A. A., Sharifi M. A. and Mulder N. J., Likelihood Based Image Segmentation and Classification: A Framework for the Integration of Expert Knowledge in Image Classification Procedures, JAG, Vol. 2, Issue 2, 2000, pp.104-118.

16- Marceau D. J. Howarth P. J., Dubois J. M. and Gratton D. J., Evaluation of the Grey-level Co-occurrence Matrix Method for Land-Cover Classification using SOPT Imagery, IEEE Transaction 
on Geoscience and Remote Sensing, Vol. 28, No. 4, 1990, pp.513518.

17- Tso B. and Mather P. M., Classification Methods for Remote Sensed Data, Taylor and Francis Inc, 2001.

18- Al-Shumam T. A. K., An Integrated Software For Remotely Sensed Data Classification, M.Sc. Thesis, University of Mosul, 2001.

Plate(1): Program of enhancement of spatial structure using texture

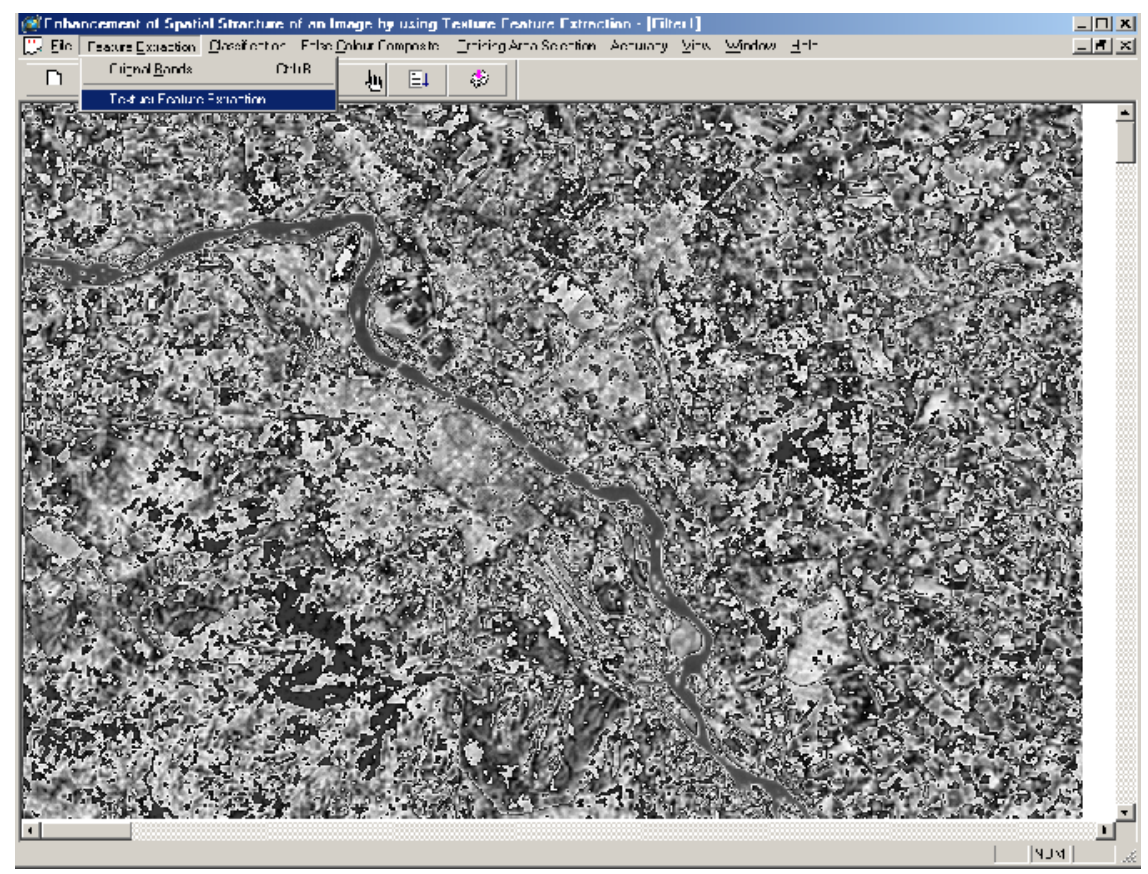


Plate(2): Original images before enhancement of spatial structure

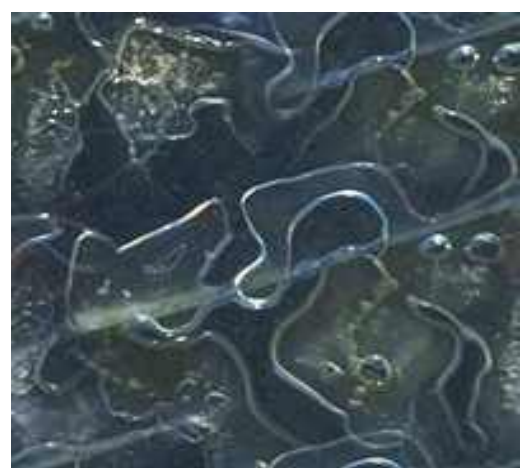

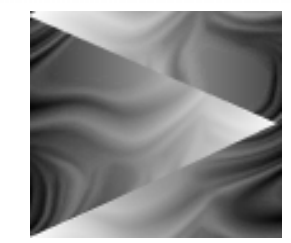

b- Fancy $(99 \times 99)$ I

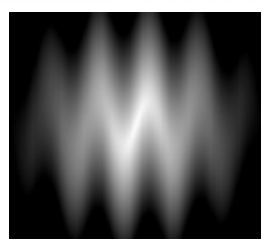

c- Wave $(99 \times 99)$ I

a- Water-Ice $(200 \times 200)$ I

Plate(3): Images after enhancement of spatial structure

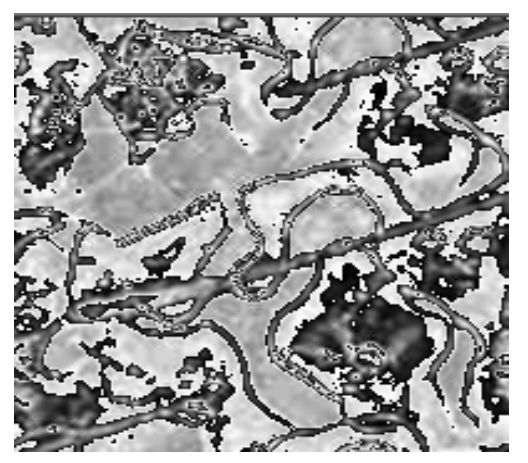

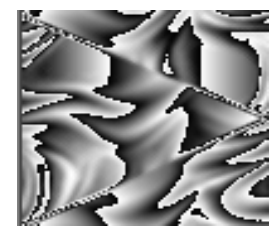

b- Fancy $(99 \times 99)$ I

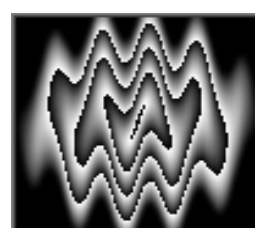

c- Wave $(99 \times 99) \mathrm{p}$

a-Water-Ice $(200 \times 200)$ I

Plate(4): TM-Sensor Row images of Mosul area and FCC's of original bands

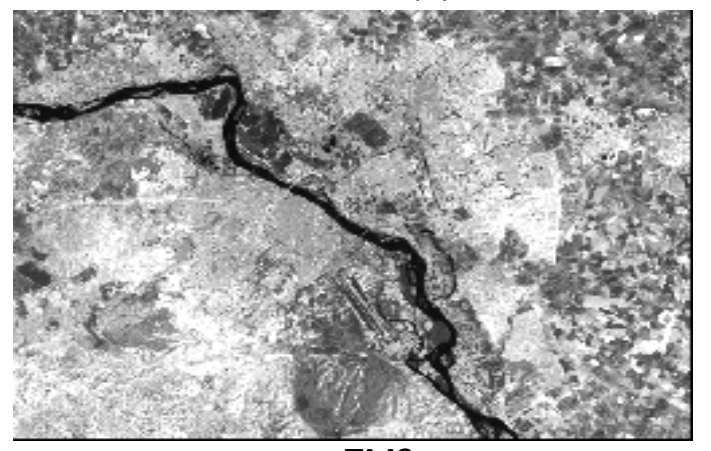

a-TM3

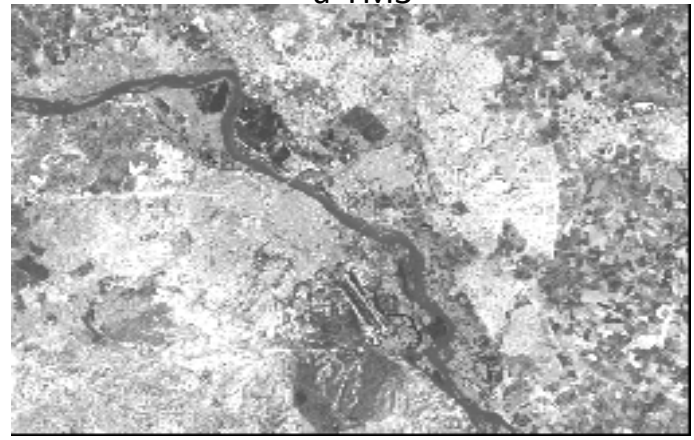

c-TM7

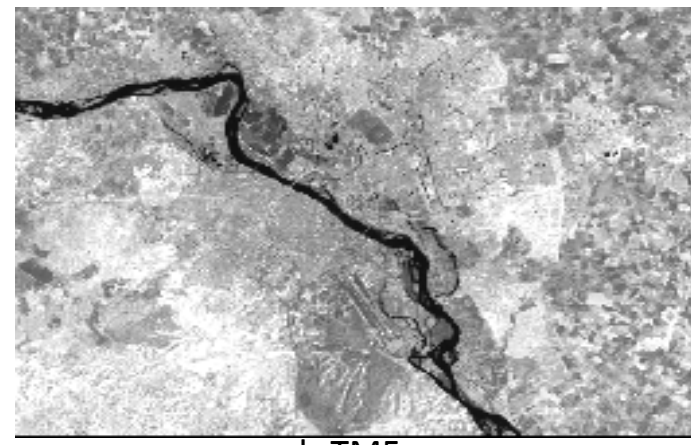

b-TM5

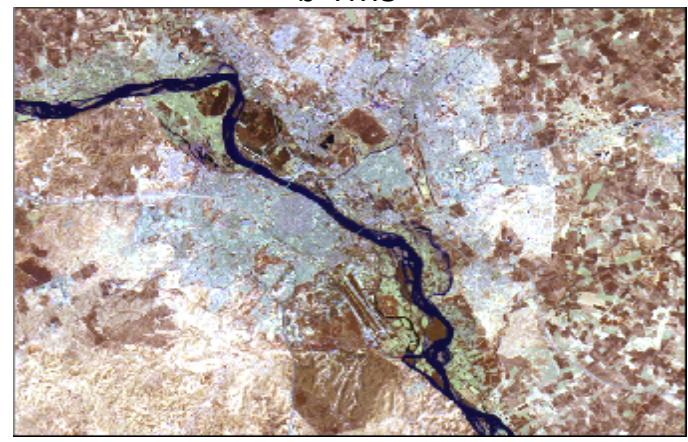

d-FCC's of original bands $(3,5,7)$ 
Plate(5): Texture feature extraction result

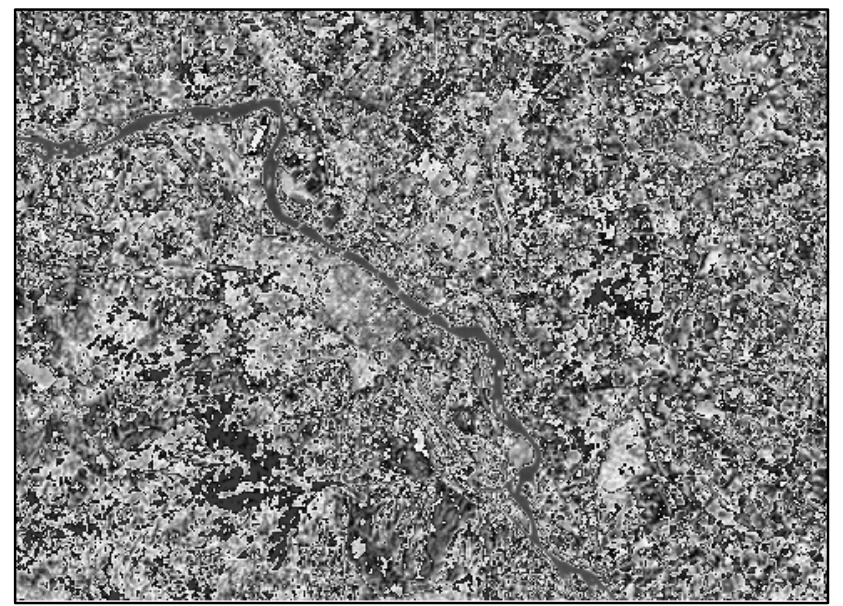

Plate(6): Maximum likelihood Classification Results
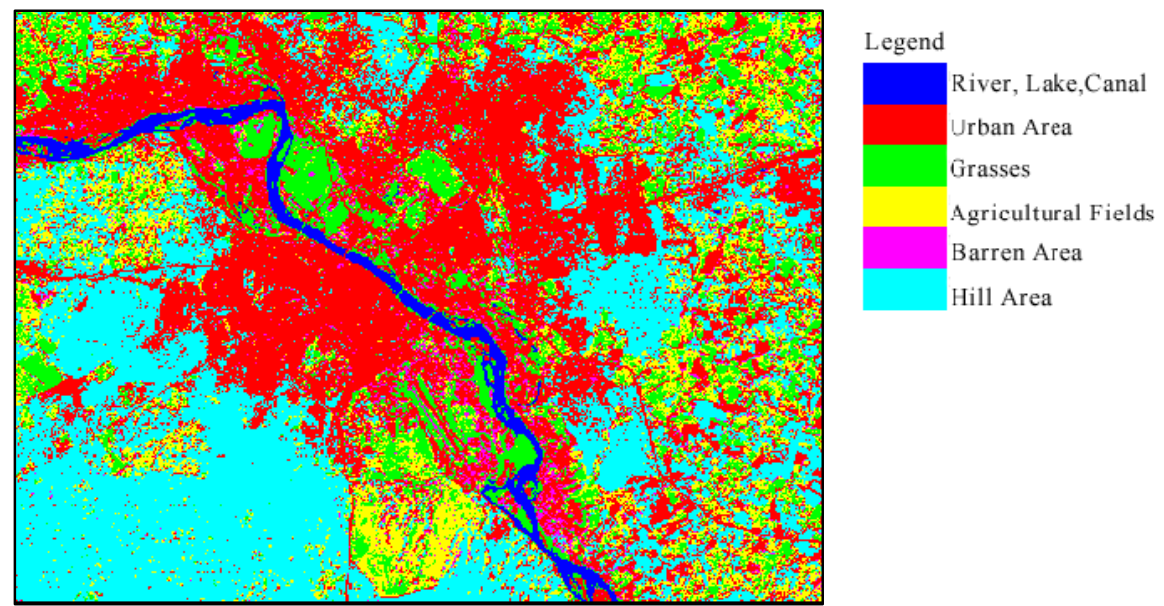

a- Original bands classification results
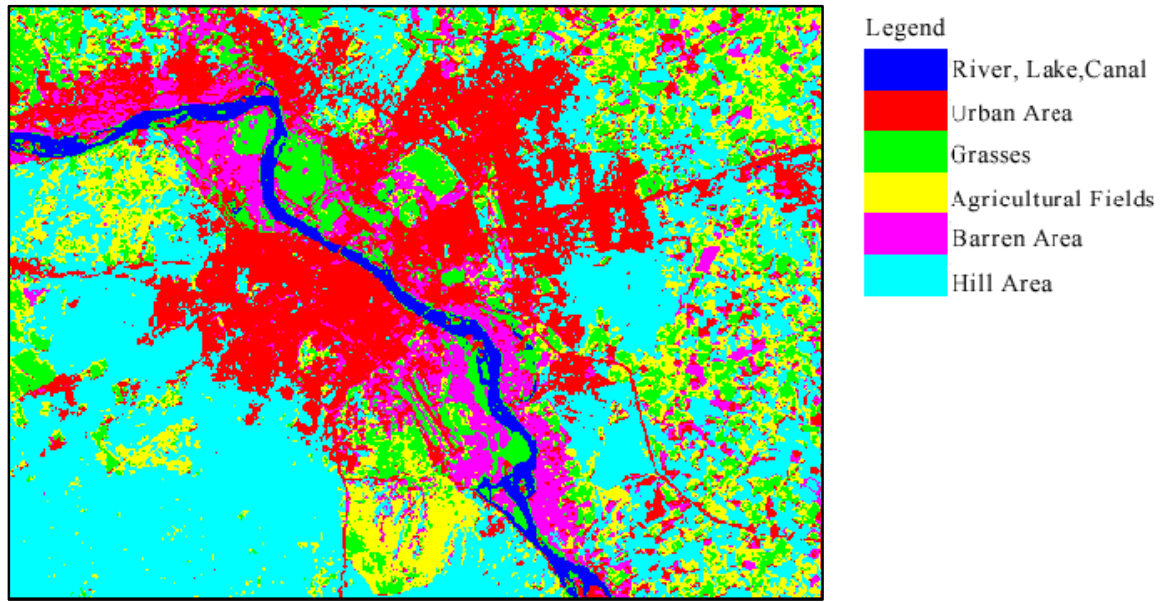

b- Texture feature extraction classification results 